\title{
Neuer Leitbegriff auf dem Weg zu einem nachhaltigen Wirtschaften?
}

\author{
Im Begriff der Green Economy bündeln sich \\ vielfälttige Vorstellungen: Vereinbarkeit von \\ Umweltschutz und Wachstum oder gar die Hoff- \\ nung auf ein nachhaltiges Wirtschaftswunder. \\ Gelingt mit der Green Economy tatsächlich der \\ Durchbruch für mehr nachhaltige Entwicklung? \\ Von Ralf Isenmann
}

$I^{\mathrm{m}}$ m Bericht „Towards a Green Economy. Pathways to Sustainable Development and Poverty Eradication“ des Umweltprogramms der Vereinten Nationen (UNEP) sind die konzeptionellen Umrisse skizziert: Green Economy zielt auf eine Art des Wirtschaftens, die das „menschliche Wohlergehen steigert und soziale Gleichheit sicherstellt, während gleichzeitig Umweltrisiken verringert und die Knappheit ökologischer Ressourcen berücksichtigt werden“ (übersetzt aus: UNEP 2011a: 2). Green Economy wird als Meilenstein auf dem Weg zu einer nachhaltigen Entwicklung verstanden, nicht als deren Ersetzung. Dazu beschreibt UNEP (2011b) elf prioritäre Handlungsfelder, darunter: Landwirtschaft, Fischerei, Wasser, Wald (Teil 1: Investition in Naturkapital), erneuerbare Energien, Produktion, Abfall, Gebäude, Transport, Tourismus und Städte (Teil 2: Investition in Energie- und Ressourceneffizienz). Zu den Handlungsfeldern bietet der Bericht zudem Hinweise zu Umsetzungsmaßnahmen sowie deren Finanzierung (Teil 3).

Die Grundidee zur Green Economy erscheint konzeptionell nicht neu, sondern ist bereits in den Ergebnisdokumenten der UNO-Konferenz über Umwelt und Entwicklung 1992 in Rio de Janeiro enthalten. Seitdem gilt Nachhaltigkeit als die Zielidee für eine langfristig ausgelegte und global lebenswerte $\mathrm{Zu}$ kunft.

Die überragende Bedeutung von Nachhaltigkeit ist in ihrem Charakter als regulative Idee zu sehen, wonach jedes Handeln so organisiert werden soll, dass es nicht auf Kosten unbeteiligter Dritter, zukünftiger Generationen oder der Natur geht. Mit anderen Worten, die drei Kriterien der Human-, Sozial- und Naturverträglichkeit sind bei allen Handlungen zu bedenken (Zwierlein/Isenmann 1995). Dies soll für alle Handlungsformen und alle gesellschaftlichen Akteure gelten. Ihr Charakter als regulative Idee verdeutlicht, dass Nachhaltigkeit einen zukunftsoffenen Such-, Lern- und Gestaltungsprozess darstellt: Orientierung stiftend, aber keinen Endzustand markierend.

\section{UNEP-Berichte als Treiber}

Der Bedeutungsaufschwung von Green Economy dürfte in der Verbreitung der oben genannten UNEP-Berichte liegen. So wurde Green Economy international auf der „Rio+20-Konferenz" forciert. Seinen Niederschlag in Deutschland hat Green Economy beispielsweise in einer Konferenz im Herbst 2012 gefunden. Unter der Leitung des Bundesministeriums für Bildung und Forschung (BMBF) und des Umweltbundesamtes (UBA) haben rund 450 Fachleute aus Wissenschaft, Wirtschaft, Politik, Verbänden und Zivilgesellschaft konferiert.

Diese Konferenz gab den Impuls für ein neues Forschungsprogramm „Green Economy“, das Anfang 2014 erwartet wird und in einen Multi-Stakeholder-Agenda-Prozess eingebettet ist. Auf politischer Bühne haben die Fraktionen CDU/CSU, SPD, FDP und BÜNDNIS 90/DIE GRÜNEN Green Economy im Deutschen Bundestag aufgegriffen (2011) und einen Umsetzungsplan: „Green Economy Roadmap“ mit einem Planungshorizont bis 2025 angestrebt (zu Roadmaps: Möhrle et al. 2013).

\section{Ein Konzept - mehrere Sichtweisen}

Die verschiedenen „Bilder“ zur Green Economy illustrieren, dass Green Economy nicht einförmig aufgegriffen wird (BMBF 2013; VDI 2013; FFU 2012). So weichen Ziele, Ansatzpunkte und Voraussetzungen voneinander ab. Ungeachtet der Schwerpunkte, die einzelne Akteure setzen, scheint ein zentraler Aspekt vergleichsweise wenig beleuchtet: die stoffliche Herausforderung einer Green Economy mit einer Steuerung und gegebenenfalls Begrenzung des quantitativen und qualitativen Stoffumsatzes.

Wie kann zum Beispiel eine Green Economy der Zielidee einer nachhaltigen Entwicklung dienen, ohne stoffliche Ressourcenziele, Ökosystemziele zur Tragfähigkeit und Biodiversitätsziele zu setzen und durch geeignete Mess- und Prüfmechanismen auszustatten? Wie werden Zielkonflikte gehandhabt? Wären nicht absolute Obergrenzen zum Ressourcen- und Energieverbrauch zu bedenken?

Auf solche noch weithin weißen Flecke macht das Autorenteam am Forschungszentrum für Umweltpolitik (FFU) in seiner Synopse zu aktuellen Wohlfahrtsansätzen und grünen Wachstumskonzepten aufmerksam (FFU 2012: 34-42). Die kritische Würdigung gibt Anlass, hinter das Konzept zu schauen und die mit Green Economy einhergehenden Forschungsfragen anzusprechen. 


\section{Forschungsfragen zu Green Economy}

Für eine strukturierte Auseinandersetzung sei hier vorgeschlagen, Forschungsfragen an eine vereinheitlichende Architektur mit vier Ebenen anzulehnen. Eine solche Architektur bietet ein Ordnungsschema, wie ein Forschungs- und Handlungsfeld aufgebaut ist, wo seine Besonderheiten liegen und wo es anschlussfähig erscheint (Isenmann 2008):

- Die Aussagenebene (context of statements) bezieht sich auf mündliche oder schriftliche Äußerungen. Sie umfasst das für ein Forschungs- und Handlungsfeld relevante Wissen in Form von Feststellungen, Behauptungen, Urteilen und Prognosen über untersuchte Tatsachen und Fakten sowie über die Basis-, Methoden- und Objektebene.

- Die Objektebene (context of phenomena) enthält die Untersuchungsgegenstände, Analyseobjekte, betrachteten Themen und -ausschnitte, relevanten Forschungsaspekte und Handlungsfelder, die behandelt werden, darunter beispielsweise Produkte, Dienstleistungen, Prozesse, Abfall, Emissionen, Stoff- und Energieströme, Umgang mit Information, Raum und anderen Ressourcen.

- Die Methodenebene (context of methods) bündelt die für ein Forschungs- und Handlungsfeld als zulässig erachteten Operationen und Untersuchungsmöglichkeiten, also die einzusetzenden Instrumente, die anzuwendenden Prinzipien, Techniken und Werkzeuge.

1 Die Basisebene (context of basics) enthält die theoretischen Grundannahmen, Eingangsvoraussetzungen, Überzeugungen, Prämissen, Axiome und Postulate, die zusammen den Charakter und die grundsätzliche Herangehensweise in einem Forschungs- und Handlungsfeld prägen.

Ihre Grundlage hat die hier herangezogene Architektur in Wissenschaftstheorie (Zwierlein 1994) und -soziologie (Krüger 1987). Die strukturgebenden Ebenen lassen sich weiter zurückverfolgen bis zur Philosophie (Rickert 1926 [1986]) und in die Wirtschaftswissenschaften (Amonn 1927).

Analog zum oben genannten Ordnungsschema seien beispielhaft Forschungsfragen formuliert, die sich mit Green Economy ergeben mögen:

- Welche neuen Handlungsoptionen und Gestaltungsempfehlungen kann Green Economy liefern, insbesondere in punkto Stoffstrommanagement?

- Welche charakteristischen Handlungsfelder sind primär zu bearbeiten? Bilden die elf im UNEP-Bericht (2011a) genannten tatsächlich die Schlüsselbereiche ab?

- Welche spezifischen Instrumente und Werkzeuge einer Green Economy können helfen, die Zielidee einer nachhaltigen Entwicklung zu befördern?

- Welche nachhaltigkeitsstrategische Ausrichtung wird mit einer Green Economy favorisiert: Effizienz, Konsistenz, Suffizienz? Welche Ausgleichsmechanismen werden vorgeschlagen? Bei den Grundannahmen wären z. B. Menschenbild, Naturverständnis sowie die Rolle von Wissenschaft, Technik und Wirtschaft inklusive Wachstumsmodelle zu reflektie- ren. Denn ein bedeutsamer konzeptioneller Kern zur Green Economy liegt darin, dass bislang unumstrittene Grundannahmen ausdrücklich thematisiert werden: Zum einen der als unausweichlich angenommene Gegensatz zwischen Umweltschutz und Wirtschaftswachstum, zum anderen die Sichtweise, dass Green Economy eine Luxusoption sei für wohlhabende Staaten und keine Ansatzpunkte für ein generelles Umdenken liefern könne (UNEP 2011a, 2-3).

Solche und ähnliche Forschungsfragen stellen sich, wenn die verschiedenen Vorstellungen zur Green Economy mit anderen stoffbezogenen Leitbegriffen wie beispielsweise Industrial Ecology (von Hauff et al. 2012; von Gleich und GößlingReisemann 2008; Isenmann und Hauff 2007) konzeptionell verglichen und auf Praxistauglichkeit untersucht werden.

\section{Green Economy und Industrial Ecology}

Aus einem Vergleich zwischen Green Economy und Industrial Ecology ergeben sich interessante Parallelen:

- Dies betrifft zum Beispiel die Rückgebundenheit der Ökonomie auf die sie umgebende Natur und die stofflich-energetische Dimension des Wirtschaftens. So beschreibt White (1994) die Industrial Ecology als „the study of the flows of materials and energy in industrial and consumer activities, of the effects of this flows on the environment, and of the influences of economic, political, regulatory, and social factors on the flow, use and transformation of resources". Insofern geht es in der Industrial Ecology darum, alle Stoffströme und Energieflüsse im Sinne eines industriellen Metabolismus zu berücksichtigen.

- Neben der Stoffbezogenheit gibt es Überschneidungen bei den Objekten. Für Green Economy und Industrial Ecology spielen beispielsweise der Aufbau von Kreislaufwirtschaftskonzepten, lokale Unternehmensverbünde, regionale Verwertungsnetzwerke, nachhaltige Industrie- und Gewerbegebiete sowie Industrial-Symbiosis-Projekte eine große Rolle.

- Bei den Methoden sind Verknüpfungen in den gerade für die Industrial Ecology typischen Instrumenten wie Lebenszyklusanalyse, Material- und Energieflussanalyse, Cradle-to-CradleDesign sowie dynamische System-Modellierung zu sehen.

- Indem die Natur und ihre ökologischen Ressourcen in die ökonomische Wert- und Schadschöpfung mit eingehen, wird sich die Ökonomie auf der Ebene der Grundannahmen mehr und mehr im Austausch mit dem und als Teil des sie umfassenden Ökosystems begreifen. Das Minimum in der Natur samt ihren ökologischen Ressourcen substanziell zu wahren, scheint darin zu bestehen, sie zugleich in der Sphäre der Ökonomie zur Anwendung zu bringen, als deren notwendige, wenn auch nicht hinreichende Basis sie damit anerkannt wäre. Dies eröffnet die Option, die enge Sichtweise der Natur als „Sack von Ressourcen“ (Hampicke 1977: 622) zu erweitern und von ihr als vorbildlich erachteter Innovationsquelle zu lernen: von ihren smarten Phänomenen, den evolutionär erprobten Strategien im Umgang 
mit Stoff, Energie, Information, Raum und Zeit sowie ihren funktionalen Grundprinzipien (Isenmann 2003).

Mit den angedeuteten Querbeziehungen, so wie sie hier am Beispiel der Industrial Ecology skizziert und durch Forschungsfragen hinterlegt sind, ließe sich zweierlei erreichen. Zum einen wäre einer Green Economy der Boden für eine konzeptionelle Anschlussfähigkeit gelegt. Zum anderen könnten die stoff- und energiebezogenen Charakteristika des Wirtschaftens in einer Green Economy als integraler Bestandteil aufgehoben sein.

$\mathrm{Ob}$ die Hoffnung auf ein nachhaltiges Wirtschaftswunder durch Green Economy insgesamt berechtigt ist? Mit dem neuen Forschungsprogramm und dem begleitenden AgendaProzess wird sich erweisen, ob und wie weit die Tür für mehr nachhaltige Entwicklung geöffnet sein mag.

\section{Anmerkung}

Der Artikel basiert auf Ergebnissen des BMBF-Projekts „Für die Zukunft gerüstet“ (Förderkennzeichen: 01PL11025).

\section{Literatur}

Amonn, A. (1927): Objekt und Grundbegriffe der theoretischen Nationalökonomie. Leipzig, Wien, Franz Deuticke.

BMBF (2012): Green Economy - Ein neues Wirtschaftswunder.

BMBF (2013): FONA - Forschung für Nachhaltige Entwicklungen - Green Economy. http://www.fona.de/green-economy.

Deutscher Bundestag (2011): Antrag der Fraktionen CDU/CSU, SPD, FDP, BÜNDNIS 90/DIE GRÜNEN - VN-Konferenz Rio+20: Nachhaltigkeit Global Umsetzen. Drucksache: 17/7182 vom 28.09.2011.

Forschungszentrum für Umweltpolitik (FFU) (Hrsg.) (2012): Synopse aktuell diskutierter Wohlfahrtsansätze und grüner Wachstumskonzepte. Erstellt von R. Zieschank et al. Freie Universität Berlin.

Gleich, A. von / Gößling-Reisemann, S. (Hrsg.) (2008): Industrial Ecology. Stuttgart, Teubner.

Hampicke, U. (1977): Landwirtschaft und Umwelt. Ökologische und ökonomische Aspekte einer rationalen Umweltstrategie, dargestellt am Beispiel der Landwirtschaft der Bundesrepublik Deutschland. Berlin.

Hauff, M. von / Isenmann, R. / Müller-Christ, G. (Hrsg.) (2012): Industrial
Ecology Management. Nachhaltige Zukunftsstrategien für Unternehmensverbünde. Wiesbaden, Springer-Gabler.

Isenmann, R. (2003): Natur als Vorbild. Plädoyer für ein differenziertes und erweitertes Verständnis der Natur in der Ökonomie. Marburg, Metropolis.

Isenmann, R. (2008): Industrial Ecology auf dem Weg zur Wissenschaft der Nachhaltigkeit? In: Gleich, A. von / Gößling-Reisemann, S. (Hrsg.): Industrial Ecology - Erfolgreiche Wege zu nachhaltigen industriellen Systemen. Stuttgart, Teubner, S. 304-315.

Isenmann, R. / Hauff, M. von (Hrsg.) (2007): Industrial Ecology. Mit Ökologie nachhaltig wirtschaften. München, Elsevier Spektrum.

Krüger, L. (1987): Einheit der Welt - Vielheit der Wissenschaften. In: Kocka, J. (Hrsg.): Interdisziplinarität: Praxis - Herausforderung - Ideologie. Frankfurt am Main, Suhrkamp, S. 106-125.

Möhrle, M. G. / Isenmann, R. / Phaal, R. (Hrsg.) (2013): Technology Roadmapping for Strategy and Innovation. Charting the Route to Success. Berlin et al., Springer.

Rickert, H. (1986): Kulturwissenschaft und Naturwissenschaft. Stuttgart, Reclam [Erstveröffentlichung Tübingen 1926].

UNEP (2011): Towards a Green Economy. Pathways to Sustainable Development and Poverty Eradication. A Synthesis for Policy Makers.

VDI Nachrichten (2013): An Green Economy kommt künftig keiner mehr vorbei. Ingenieur.de, 23. 05. 2013.

White, R. (1994): Preface. In: Allenby, B. R. / Richards, D. J. (Hrsg.): The Greening of Industrial Ecosystems. Washington, Nat. Ac. Press.

Zwierlein, E. (1994): The Paradigm of Self-Organization and its Philosophical Foundations. In: Mishra, R. K. et al. (Hrsg.): On Self-Organization. An Interdisciplinary Search for a Unifying Principle. Berlin et al., Springer, S. 288-298.

Zwierlein, E. / Isenmann, R. (1995): Ökologischer Strukturwandel und Kreislaufökonomie. Wege zu einer umweltorientierten Materialwirtschaft. Idstein, Schulz-Kirchner.

\section{AUTOR + KONTAKT}

Dr. habil. Ralf Isenmann ist Professor für Nachhaltiges Zukunftsmanagement an der Hochschule München.

Hochschule München, Fakultät 10 Betriebswirtschaft, Am Stadtpark 20, 81243 München. Tel.: +49-89-1265-2757, E-Mail: isenmann@hm.edu, Website: www.bwl.hm.edu

\section{Nachhaltigkeit}

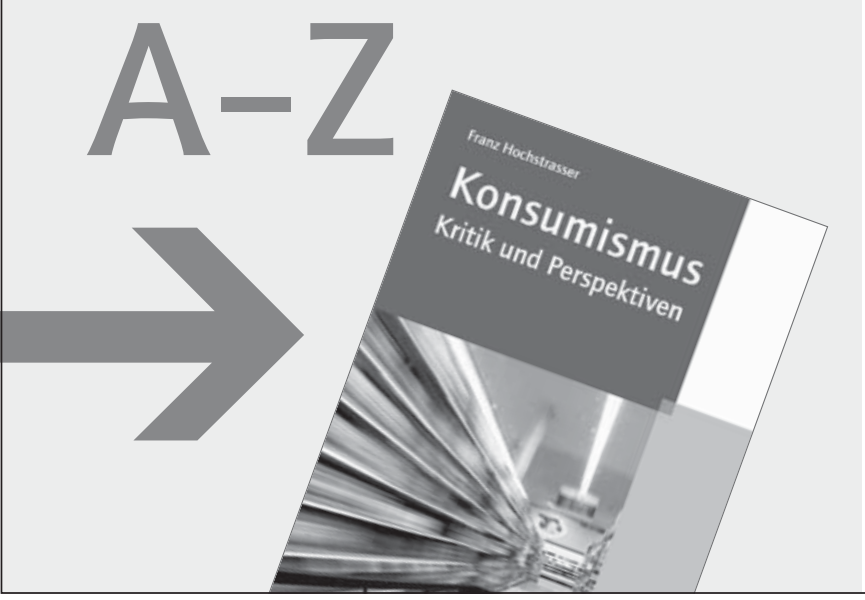

\section{A wie Alternative}

Weltweit definieren sich die Menschen zunehmend über den Konsum. So hat sich eine weit verbreitete konsumistische Kultur entwickelt, die eng mit unserem Wirtschaftssystem verwoben ist. Dies ist aber keineswegs schicksalhaft. Alternativen zum Konsumismus sind möglich - außerhalb und auch innerhalb etablierter Strukturen und Lebenswelten.

\section{F. Hochstrasser}

Konsumismus

Kritik und Perspektiven

364 Seiten, broschiert, 19,95 Euro,

ISBN 978-3-86581-326-8

\section{Erhältlich bei}

www.oekom.de | oekom@verlegerdienst.de

Die guten Seiten der Zukunft 
Copyright (C) 2013, IÖW und oekom Verlag. Die Nutzung des Artikels ist Abonnenten von Ökologisches Wirtschaften vorbehalten. Nachdruck und Vervielfältigung des Artikels einschließlich Speicherung und Nutzung auf optischen und elektronischen Datenträgern nur mit Zustimmung der Redaktion von Ökologisches Wirtschaften (http://www.oekologischeswirtschaften.de). 